\title{
Enfermería enfocada en el Síndrome de Sjögren
}

\author{
Dry mouth syndrome in the elderly
}

\author{
Rocío B. Mayorga-Ponce ${ }^{a}$ y Brenda A. González Monter ${ }^{b}$
}

\begin{abstract}
:
Sjögren's Syndrome is an autoimmune disease that affects various systems of the human body, primarily its exocrine glands. Patients suffering from this disease have significantly compromised their quality of life, in older adults it is considerably associated with mental disorders such as depression. A review of the literature of various articles published in journals around the world was carried out to inform the population of the generalities of the disease, as well as the signs and symptoms of Sjögren's Syndrome, the main complications and the known treatment to improve the quality of life of the elderly.
\end{abstract}

Keywords:

Sjögren's Syndrome, autoinmmune disease, quality of life

Resumen:

El Síndrome de Sjögren es una enfermedad autoimmune que afecta varios sistemas del organismo humano, primordialmente las glándulas exocrinas de éste. Los pacientes que padecen esta enfermedad tienen de manera significativa comprometida su calidad de vida, en los adultos mayores se asocia considerablemente a trastornos mentales tales como la depresión. Se realizó una revisión de la literatura, de diversos artículos publicados en revistas de todo el mundo, para dar a conocer a la población las generalidades de la enfermedad, así como los signos y síntomas del Síndrome de Sjögren, las diversas manifestaciones y el tratamiento conocido para mejorar la calidad de vida del adulto mayor.

Palabras Clave:

Síndrome de Sjögren, enfermedad autoimmune, calidad de vida

\section{Introducción}

Cuando llega la vida adulta, la calidad de vida comienza a verse comprometida debido a diversas enfermedades, en diferentes aspectos, el aspecto funcional, el mental, el clínico, y el socio-familiar. Una de las enfermedades que afecta al adulto mayor de manera múltisitemica es el síndrome de Sjögren (SS). Los pacientes que padecen esta enfermedad se ven afectados de manera considerable ya que las glándulas exocrinas de su organismo dejan de funcionar como deberían. La afectación se manifiesta fundamentalmente por la aparición de xeroftalmía (sequedad en los ojos) y xerostomía (sequedad en la boca), ocasionando la grave falta de lubricación en los ojos por la ausencia de lágrimas, lo que a la larga puede generar lesiones en el epitelio ocular, también encontramos problemas al deglutir los alimentos por la escases de producción salival en la boca. [1]

Cabe mencionar que sólo el $30 \%$ de los pacientes que padecen Sjögren presentan otro tipo de síntomas, entre los que se encuentran los musculoesqueléticos, genitourinarios, neurológicos, cutáneos, renales y gastrointestinales ${ }^{[2]}$. Sin embargo, el SS está muy relacionado con las enfermedades reumáticas, dado que puede generar síntomas comunes, como los dolores o las inflamaciones de las articulaciones (artritis). ${ }^{[3]}$

Autor de Correspondencia, Universidad Autónoma del Estado de Hidalgo, https://orcid.org/0000-0003-3544-7171, Email: rmponce@uaeh.edu.mx

${ }^{\mathrm{b}}$ Universidad Autónoma del Estado de Hidalgo, https://orcid.org/0000-0002-3903-2590, Email: go262777@uaeh.edu.mx 


\section{¿Qué es un Síndrome de Sjögren primario y secundario?}

Cuando el paciente presenta SS de manera aislada, es decir que se define con sequedad en ojos y boca sin ningún otro trastorno autoinmunitario, lo llamamos SS PRIMARIO. De tal manera que el SS SECUNDARIO ocurre solo cuando se asocia al paciente con diferentes enfermedades autoinmunitarias cómo artritis reumatoidea, lupus eritematoso sistémico, dermatomiositis o fibromialgia. ${ }^{[4]}$

\section{Figura 1. Tratamiento sintomático para paciente con Síndrome de Sjögren.}

Fuente: Elaboración propia.

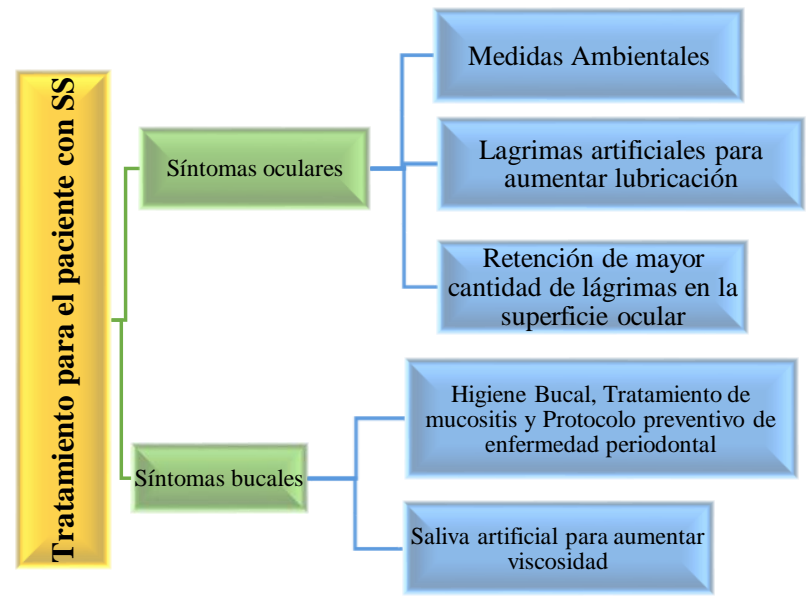

El tratamiento para el SS primario, tal como se muestra en la Figura 1, se complementa con estimulantes de secreción sistémica, como agentes muscarínicos y en los casos de SS secundario entra la participación de antiinflamatorios tópicos, anti-inflamatorios sistémicos e inmuno-moduladores. ${ }^{[5]}$

\section{La importancia de la enfermería aplicada en el Síndrome de Sjögren}

El cuidado de los pacientes y su calidad de vida es la principal esencia de los profesionales en enfermería, es por ello que siguiendo esta premisa el plan de cuidados para el paciente con SS va encaminado a la conservación, la reeducación, el restablecimiento y el autocuidado de la vida, esto fundamentado en la relación terapéutica enfermero/a-paciente. ${ }^{[6]}$

La promoción de la Salud es el arma con la que el profesional de enfermería va a actuar, principalmente educando a su paciente sobre la higiene bucal, lavado matutino de ambos ojos, fomento de la abundante ingesta líquida y la adecuada ingesta de alimentos sólidos para facilitar la deglución. Los cuidados enfermeros se complementan con las medidas preventivas necesarias para evitar la complicación de las enfermedades multisistémicas ya adquiridas, cómo la administración de lágrimas y saliva artificiales, promover evitar la ingesta de alimentos secos y erosivos, realizar un programa de entrenamiento funcional; que puede incluir la amplitud del movimiento, fortalecimiento muscular y resistencia, y la aplicación de calor o frío en las articulaciones. ${ }^{[7]}$

De manera general se trabaja para satisfacer y reeducar al paciente a fortalecer las AVD (actividades de la vida diaria) apoyándose en el profesional de terapias ocupacionales específicamente dirigidas al paciente que lo necesita.

\section{Referencias}

[1] Riega J, Villarreal A, Ceceñas L, Salas J. Síndrome de Sjögren (SS), revisión del tema y saliva como método diagnóstico. Gac Med Mex [Internet]. 2016 [Citado el 22 de octubre 2021]; 152:371-80. Recuperado de: https://www.anmm.org.mx/GMM/2016/n3/GMM_152_2016_3_371380.pdf

[2] Camino J, Vásquez M, Fernández F, Peralta C. Manifestaciones extraglandulares renales del síndrome de Sjögren. Revista Cubana de Reumatología [Internet]. 2017 [Citado el 22 de octubre 2021]; 19(3):126-131. Recuperado de: https://www.redalyc.org/articulo.oa?id=451653250004

[3] Cañas C. Síndrome Seco - Síndrome de Sjögren. Carta de la Salud [Internet]. 2002 [Citado el 22 de octubre 2021]; 74. Recuperado de: https://repository.icesi.edu.co/biblioteca_digital/bitstream/10906/4507 /1/074_sindrome_seco_sindrome_sjogren.pdf

[4] Diez C, Lema J, Álvarez N, Atanes A, De Toro F, Pinto J, et al. Aspectos actuales del síndrome de Sjögren: etiopatogenia, manifestaciones clínicas, diagnóstico y tratamiento. Semin Fund Esp Reumatol [Internet]. 2010 [Citado el 22 de octubre 2021]; 11(2):70-76. Recuperado de: https://www.elsevier.es/es-revista-seminariosfundacion-espanola-reumatologia-274-articulo-aspectos-actuales-delsindrome-sjogren-S1577356610000230

[5] Muñoz E, Tobón G. Síndrome Seco. Carta de la Salud [Internet]. 2013 [Citado el 22 de octubre 2021]; 203. Recuperado de: https://valledellili.org/wp-content/uploads/2018/04/pdf-203cartadelasalud-abril2013-1.pdf

[6] Juárez P, García M. La importancia del cuidado de enfermería. Rev Enferm Inst Mex Seguro Soc [Internet]. 2009 [Citado el 22 de octubre 2021]; $17 \quad$ (2): 109-111. Recuperado de: http://revistaenfermeria.imss.gob.mx/editorial/index.php/revista_enfer meria/article/view/498/488

[7] Arévalo Elena, Domínguez S, González I. Cuidados de Enfermería en pacientes con xerostomía [Internet]. España: Revista Electrónica de Portales Medicos; 2015. [Citado el 22 de octubre 2021]. Recuperado de: https://www.revista-portalesmedicos.com/revistamedica/cuidados-de-enfermeria-xerostomia/ 